TGA-TAA). We confirmed by SDS-polyacrylamide gel electrophoresis (PAGE) that the mutant donor plasmids express wild-type levels of LtrA protein.

16. In 29 of 39 mobility products, the group I intron spliced using the normal $5^{\prime}$ and $3^{\prime}$ splice sites, and eight used cryptic $5^{\prime}$ splice sites at Ll.LtrB positions 1810 (six clones), 1985 (one clone), and 2185 (one clone).

17. PACD- $\triangle O R F+O R F 1$ (Fig. 1D) is a derivative of PACD$\triangle \mathrm{ORF}(15)$. The donor intron has a deletion in the loop of intron domain IV, which removes most of the LtrA ORF, but retains regions of domain IV that are required for binding the LtrA protein [H. Wank, J. San Filippo, R. N. Singh, M. Matsuura, A. M. Lambowitz, Mol. Cell 4, 239 (1999)]. An intact LtrA ORF was cloned downstream of the $3^{\prime}$ exon in the same plasmid (1.9-kb Sca I-Sal I fragment of pACD-LtrB filled in with Klenow polymerase inserted into the Fsp I site). PACD- $\triangle$ ORF + ORF2 is a derivative of PACD- $\triangle$ ORF+ORF1 that contains shorter exons (26 bp of E1 preceded by a Hind III site and 11 bp of E2 followed by a Pst I site) to facilitate manipulation of the IBS and $\delta^{\prime}$ sequences.

18. Reverse splicing assays showed that RNP particles from cells expressing PACD- $\triangle O R F+O R F 1$ ( $\triangle$ ORF intron) insert intact intron RNA, whereas those from cells expressing PACD-LtrB (full-length intron) insert partially degraded intron RNA (5). SDS-PAGE showed that $\mathrm{PACD}-\triangle \mathrm{ORF}+\mathrm{ORF} 1$ and $\mathrm{pACD}$-LtrB express similar levels of LtrA protein.

19. For supplementary data, see Science Online (www. sciencemag.org/feature/data/1050641.shl).

20. G. Mohr, X. Cui, A. M. Lambowitz, unpublished data.

21. M. Dean et al., Science 273, 1856 (1996).

22. R. Liu et al., Cell 86, 367 (1996).

23. S. J. Deminoff, J. Tarnow, G. M. Santangelo, Genetics 141, 1263 (1995).

24. Recipient plasmids containing HIV- 1 and CCR 5 target sites were constructed by inserting the indicated segments of the target DNA into the Xho I site of the polylinker of pBRR-Tet (13) by blunt-end ligation after filling in sites with T4 DNA polymerase. Target DNAs were inserted in both the sense and antisense orientations relative to the tet ${ }^{R}$ gene. HIV-1 and CCR5 target DNAs were derived from PLAI3 [K. Peden, M. Emerman, L. Montagnier, Virology 185, 661 (1991)] and PCMV-CKR5 [P. Bieniasz et al., EMBO J. 16,2599 (1997)], respectively. For targeting experiments, the donor plasmid pool was electroporated into cells containing the desired recipient plasmid.

25. The combinatorial intron library PACD-DL was constructed in PACD- $\triangle O R F+O R F 2$ (17) by inserting random sequences in place of EBS and $\delta$ positions (EBS2, -13 to -8 ; EBS1, -6 to -1 ; and $\delta,+1$ to +4 ) that potentially form base pairs with the DNA target site. The corresponding $5^{\prime}$ exon positions in IBS1 and IBS2 of the precursor RNA were also randomized to provide nucleotide combinations that could form base pairs with the randomized EBS sequences for RNA splicing. The randomized sequences were introduced in a two-step PCR, in which the first step used primers that contain the randomized sequences to generate two partially overlapping PCR products (positions -26 to 222 and 202 to 318). The two PCR products were mixed and amplified with external primers, which introduce Hind III and Bsr Gl sites, and then cloned between the corresponding sites of PACD- $\triangle O R F+O R F 2$. The ligation products were electroporated into $E$. coli $\mathrm{DH} 10 \mathrm{~B}$, and the library was amplified in LB medium containing chloramphenicol.

26. For selection experiments, the donor plasmid library containing $\sim 2 \times 10^{8}$ different introns was transformed into $E$. coli HMS174(DE3) containing recipient plasmids with HIV-1 or CCR5 DNAs inserted upstream of the promoterless tet ${ }^{R}$ gene [PBRRHIV1(1-2561a)/Tet, pBRR-HIV1(2562-5371a)/Tet, or pBRR-CCR5(s)/Tet) (24)]. About $10^{8} \mathrm{Amp}^{\mathrm{R}} \mathrm{Cam}^{\mathrm{R}}$ transformants were obtained. The frequency of Tet $^{R}$ colonies was $2.5 \times 10^{-6}$, with $40 \%$ having mobility events. The integrated introns were then reconstructed in the PACD- $\triangle$ ORF+ORF2 donor plasmid (17) by PCR amplifying a segment extending from position -13 in the target site to intron position 326 downstream of the EBS sequences, by using the $3^{\prime}$ primer
LtrBA2 in combination with a target site specific 5 primer. The latter has a $5^{\prime}$ Hind III site, followed by wild-type $5^{\prime}$ exon positions -26 to -14 , and the target site junction sequence from -13 to +18 in the intron. The PCR products were digested with Hind III and Bsr Gl, and swapped for the corresponding segment of PACD- $\triangle O R F+O R F 2$. Mobility frequencies for the selected introns were determined in the $E$. coli genetic assay using recipient plasmids containing the HIV-1 or CCR5 DNA target sites (24).

27. Human embryonic kidney 293 cells $\left(10^{6}\right)$ were transfected with the CCR5 CDNA plasmid PT7CCR5 $(1 \mu \mathrm{g})$ and/or RNP particles containing intron CCR5-332s $(1.2 \mu \mathrm{g})$ in $12 \mu \mathrm{l}$ of DMRIE-C (Gibco-BRL) for 4 hours. CEM T cells $\left(3 \times 10^{5}\right)$ were transfected with pLAlgagpol $(0.5 \mu \mathrm{g})$ and/or RNP particles containing intron HIV1-4069s $(0.5 \mu \mathrm{g})$ in $12 \mu \mathrm{l}$ of DMRIE-C for 5 hours. In both cases, DNA was incubated with half the total lipid for 15 to $30 \mathrm{~min}$, and RNPs were incubated with the remaining half just before mixing and transferring to cells, to minimize the possibility that reverse splicing occurs extracellularly. DNA was isolated from transfected cells after 24 hours using a QIAamp DNA Blood Kit (Qiagen). The RNP particles used in the transfections were reconstituted with in vitro spliced intron RNA and purified IEP, as described [R. Saldanha et al., Biochemistry 38, 9069 (1999)].
28. Nested PCR (total of 60 cycles) was carried out on DNA samples treated with ribonuclease. Upstream primers were specific to target DNA sequences (CCR5 primer, 5'GCGCGGATCCATGGATTATCAAGTGTCAAG; nested primer, 5'-CCGAAGCT TGTCAACTCT TGACAGGGCTC) (HIV-1 pol primer, GGGGGGATCCACACAAAGGAATTGGAGG; nested primer, GGGGGGATCCGTCAGTGCTGGAATCAGG). Downstream primers were specific to intron sequences (primer, $5^{\prime}$ GGCCCTTTCGT TTCGT TC; nested primer, 5'-GGCCCGATTGTCTTTAGGTA). PCR products containing the integrated CCR5 intron were cut with Rsa I at a site located in the intron, and those containing the integrated HIV-1 pol intron were cut with Bst XI at a site located in the HIV-1 pol sequence. PCR products were cloned by standard techniques and sequenced using a Thermo Sequenase Radiolabeled Cycle Sequencing kit (USB).

29. M. Karberg, H. Guo, R. Coon, A. M. Lambowitz, in preparation.

30. We thank J. Edwards for technical assistance and $M$. Belfort (Wadsworth Center, Albany, NY) for comments on the manuscript. Supported by NIH grants GM37949 (A.M.L.) and Al40981 (B.S.).

21 March 2000; accepted 18 May 2000

\title{
A Neural Basis for General Intelligence
}

\author{
John Duncan, ${ }^{1 *}$ Rüdiger J. Seitz, ${ }^{2}$ Jonathan Kolodny, ${ }^{1}$ \\ Daniel Bor, ${ }^{1}$ Hans Herzog, ${ }^{3}$ Ayesha Ahmed, ${ }^{1}$ Fiona N. Newell, ${ }^{1}$ \\ Hazel Emslie ${ }^{1}$
}

\begin{abstract}
Universal positive correlations between different cognitive tests motivate the concept of "general intelligence" or Spearman's $g$. Here the neural basis for $g$ is investigated by means of positron emission tomography. Spatial, verbal, and perceptuo-motor tasks with high-g involvement are compared with matched low- $g$ control tasks. In contrast to the common view that $g$ reflects a broad sample of major cognitive functions, high- $g$ tasks do not show diffuse recruitment of multiple brain regions. Instead they are associated with selective recruitment of lateral frontal cortex in one or both hemispheres. Despite very different task content in the three high- $g$-low- $g$ contrasts, lateral frontal recruitment is markedly similar in each case. Many previous experiments have shown these same frontal regions to be recruited by a broad range of different cognitive demands. The results suggest that "general intelligence" derives from a specific frontal system important in the control of diverse forms of behavior.
\end{abstract}

As discovered by Spearman (1) early in the last century, measures of performance or success in diverse cognitive tests show a pattern of almost universal positive correlation: To some extent at least, the same people tend to perform well in very different tasks. To explain this result, Spearman put forward the hypothesis of a general or $g$ factor making some contribution to success in diverse forms of cognitive activity. People with high $g$ scores will be those usually performing well, leading to the interpretation of

${ }^{1}$ Medical Research Council Cognition and Brain Sciences Unit, 15 Chaucer Road, Cambridge CB2 2EF, UK. ${ }^{2}$ Department of Neurology, Heinrich-Heine-University Düsseldorf, Moorenstrasse 5, D-40225 Düsseldorf, Germany. ${ }^{3}$ Institute of Medicine, Research Center Jülich, D-52407 Jülich, Germany.

*To whom correspondence should be addressed. Email: john.duncan@mrc-cbu.cam.ac.uk $g$ as "general intelligence." Factor analysis can be used to show which tasks are most correlated with $g$ and are thus the best general intelligence measures; often, these turn out to be tests of novel problem solving such as Raven's Progressive Matrices (2). An alternative hypothesis, originally proposed by Thomson (3), has also received detailed consideration. According to this hypothesis, any task receives contributions from a large set of component factors or information-processing functions. Universal positive correlation arises not for any common reason, but simply because any two tasks are likely to share at least some components. The " $g$ factor" measured by standard intelligence tests is now interpreted as the average efficiency of the total set of cognitive functions (4); as Thomson showed, tasks with high apparent $g$ correlations will be those sampling the total set 
Fig. 1. Example test items for each task. Each item consisted of four display elements (drawings or letter sets), and the task was to identify the element that in some sense mismatched or differed from the others. In each task, participants completed as many items as possible in a fixed period of 4 (behavioral sessions) or 2 (PET sessions) $\mathrm{min}$, after $0.5 \mathrm{~min}$ of practice. (A) Materials for the high-g spatial task were adapted with permission from a standard nonverbal test of $g$, Cattell's Culture Fair, Scale 2 Form A and Scale 3 (17). Display elements were four panels, each containing one or more shapes, symbols, or drawings. One panel differed in some respect from the others; extensive problem solving was required to identify this panel because the difference could concern any property, often abstract and/or complex. In the example shown the relevant property is symmetry; the mismatching panel is the third in the row. In the low- $g$ spatial control task, in contrast, there was minimal problem solving. In each display, the four panels each contained a single geometrical shape, three of which were physically identical whereas the fourth differed in some visually obvious respect (shape, texture, size, orientation, or a combination of these). (B) Materials for the high- $g$ verbal task were adapted with permission from a standard letter-based problem-solving task, Letter Sets from the ETS Kit of Factor-Referenced Tests (24). The high $g$ loading of the original test was established by analysis of a large preexisting data set (25). Display elements were four sets of four letters each. One set differed in some respect from the others; again, the task required extensive problem solving because a variety of alphabetic and other rules could distinguish the mismatching letter set in any given test item. In the example the mismatching set is the third, whose letters are equally spaced in the alphabet. In the low- $g$ verbal control task, the task was simply to find the one set in each display whose letters were not in strict alphabetical order. (C) Displays in the circles task were based with permission on two drawings taken from a single item of the Cattell Culture Fair (17). As illustrated, in one drawing the smallest circle was toward the center of the overall figure, whereas in the other it was toward the periphery. Again there were three identical panels (all small circles central, or all peripheral), and the task was to identify the single panel with the alternative arrangement. For all tasks, displays were presented on an Apple Macintosh monitor; horizontal display extent was approximately $12^{\circ}$ for spatial tasks, $19^{\circ}$ for verbal tasks. The position of the mismatching element was indicated by pressing the corresponding key on a four-choice keyboard, operated with middle and index fingers of the two hands. The screen cleared when a response was made, and a new test item was presented after a pause of $500 \mathrm{~ms}$. In problem-solving tasks, participants were encouraged not to guess but to work on each problem until they were confident of their answer. These arrangements ensured that participants worked continuously throughout the period of each task, despite long solution times for problemsolving items but much shorter times for control items.

of cognitive functions most broadly. The indeterminacy of factor analysis has made it impossible to distinguish these alternative hypotheses from correlational data, and after almost a century of debate, both are still vigorously defended (5). Here we use positron emission tomography (PET) to investigate the neural basis for $g$ and the light this casts on Spearman's and Thomson's interpretations.

One possibility - more closely allied to the Spearman view-is that $g$ may reflect some relatively confined set of neural functions, broadly contributing to success in diverse cognitive tests. In recent years, in particular, similarities have been noted between some effects of frontal lobe lesions and the normal behavior of people from the lower part of the $g$ distribution, suggesting that frontal functions may be particularly central to $g(6)$. Though frontal functions are not well understood - as reflected in rather general information-processing concepts such as executive control, strategy formation, or monitoring the contents of working memory (7) - certainly they are important in a wide diversity of behavior, as a major role in $g$ would imply. According to this hypothesis, tasks with high $g$ correlations should be characterized by specific recruitment of prefrontal cortex. The alternative - directly implied by the Thomson hypothesis - is that increasing $g$ correlations should be associated with an increasingly diverse pattern of neural activation, reflecting increasingly broad sampling of all major cognitive functions.

Our method took advantage of the psychometric finding that tasks with very different surface content can share the property of high $g$ correlation (8). Thus, converging investigations of several high- $g$ tasks can be used to ask what property these tasks share at the level of neural activity. In our first test, we used problemsolving tasks based on spatial and verbal materials. In each case, we began by adapting standard psychometric tests whose $g$ correlations were known to be high. Example problems from spatial and verbal tasks are shown in Fig. $1, \mathrm{~A}$ and $\mathrm{B}$. In two large- $N$ behavioral studies, we confirmed high $g$ correlations in the adapted task versions (9) (Table 1). For each task, we developed a corresponding low- $g$ control, based
Table 1. Behavioral data for all tasks. Correlations with standard measures of $g$ derive from one (spatial and circles tasks) or two (verbal tasks) behavioral studies conducted before the PET experiment (9). Correlations for verbal tasks are averages from the two studies calculated by Fisher's $z$-transform. $N$ denotes number of participants contributing to each correlation, excluding cases with missing data.

\begin{tabular}{lcc}
\hline Task & $g$ correlation $(r)$ & $N$ \\
\hline & Spatial & \\
High-g & 0.59 & 56 \\
Low-g & 0.37 & 54 \\
& Verbal & \\
High-g & 0.55 & 103 \\
Low-g & 0.41 & 102 \\
& Circles & \\
& 0.67 & 53 \\
\hline
\end{tabular}

on similar materials but without the problemsolving element. Again, behavioral pretesting confirmed the lower $g$ correlations of these newly developed control tasks. In all cases, tasks were designed to keep the participant continuously active, despite wide variations in the time taken to respond to individual test items. We then used PET to compare each high- $g$ problem-solving task with its corresponding low- $g$ control (10).

Regions of significantly greater blood flow ( $P<0.05$, corrected for multiple comparisons) in high- $g$ compared with low- $g$ tasks are shown in Fig. 2, A (spatial comparison) and B (verbal comparison) (11). Corresponding peak activations appear in Table 2. In the spatial comparison, the strongest high- $g$ activations occurred bilaterally in the lateral prefrontal cortex, and in a discrete region of the medial frontal gyrus/ anterior cingulate. Elsewhere in the brain, activations were restricted to the posterior visual system, presumably reflecting more extensive visual analysis and/or the effects of eye movements, and to discrete regions of parietal and premotor cortex, recruited in a wide range of visuospatial tasks (12). These results resemble those previously seen in comparisons of Raven's Progressive Matrices with simple sensorimotor controls (13). For the verbal comparison, the only significant high- $g$ activation occurred in the lateral frontal cortex of the left hemisphere, closely corresponding to the similar activation in the spatial comparison.

Such results argue strongly against the possibility that high- $g$ tasks are associated with diffuse neural recruitment, as predicted by broad sampling of the brain's major cognitive functions. Examination of the data at a less conservative significance threshold $(P<0.001$ uncorrected) did not change this conclusion; in the spatial comparison, there was simply a strengthening of the major activation foci shown in Fig. 2, whereas in the verbal comparison, frontal activation was accompanied by weak occipital activations resembling those 
Table 2. Peak activations for each high- $g$ minus low- $g$ task contrast. Coordinates $[x, y, z$ in space of Montreal Neurological Institute (MNI) template] and selection of foci are according to the conventions of SPM99. Brain regions (approximate Brodmann areas) are estimated from Talairach and Tournoux (23), after adjustment (www.mrc-cbu. cam.ac.uk/Imaging/mnispace.html) for differences between $\mathrm{MNI}$ and Talairach coordinates.

\begin{tabular}{|c|c|c|}
\hline Brain region & Coordinates & z score \\
\hline \multicolumn{3}{|c|}{ Spatial $(\mathrm{P}<0.05$ corrected $)$} \\
\hline \multicolumn{3}{|c|}{ L lateral frontal } \\
\hline 46 & $-52,44,4$ & 5.8 \\
\hline 46 & $-52,32,26$ & 5.2 \\
\hline 47 & $-48,32,-8$ & 4.9 \\
\hline 8 & $-34,10,40$ & 4.5 \\
\hline \multicolumn{3}{|l|}{ R lateral frontal } \\
\hline 46 & $56,30,24$ & 5.7 \\
\hline 45 & $64,20,16$ & 5.6 \\
\hline 46 & $48,44,12$ & 4.8 \\
\hline 6 & $40,12,64$ & 5.2 \\
\hline \multicolumn{3}{|l|}{$\mathrm{R}$ medial frontal } \\
\hline 8 & $2,26,46$ & 5.7 \\
\hline \multicolumn{3}{|l|}{ L parietal } \\
\hline $7 / 19$ & $-26,-66,40$ & 4.6 \\
\hline \multicolumn{3}{|l|}{$\mathrm{R}$ parietal } \\
\hline $40 / 7$ & $48,-56,50$ & 5.1 \\
\hline \multicolumn{3}{|l|}{ L occipital } \\
\hline 18 & $-24,-104,-10$ & 5.0 \\
\hline 18 & $-38,-94,-8$ & 4.8 \\
\hline \multicolumn{3}{|l|}{ R occipital } \\
\hline 18 & $40,-94,-6$ & 5.3 \\
\hline 19 & $46,-74,-12$ & 5.2 \\
\hline \multicolumn{3}{|c|}{ Verbal ( $\mathrm{P}<0.05$ corrected) } \\
\hline \multicolumn{3}{|c|}{ L lateral frontal } \\
\hline 46 & $-54,32,22$ & 5.3 \\
\hline 47 & $-50,32,-8$ & 5.2 \\
\hline $10 / 46$ & $-50,46,0$ & 5.0 \\
\hline \multicolumn{3}{|c|}{ Circles $(\mathrm{P}<0.001$ uncorrected $)$} \\
\hline \multicolumn{3}{|c|}{$\mathrm{R}$ lateral frontal } \\
\hline 46 & $56,28,26$ & 3.4 \\
\hline 9 & $44,34,36$ & 3.3 \\
\hline 6 & $38,4,50$ & 3.7 \\
\hline 6 & $44,12,62$ & 3.5 \\
\hline \multicolumn{3}{|l|}{ L occipital } \\
\hline 18 & $-24,-104,-10$ & 5.1 \\
\hline 18 & $-6,-82,2$ & 3.3 \\
\hline \multicolumn{3}{|l|}{ R occipital } \\
\hline 18 & $24,-102,-8$ & 6.4 \\
\hline
\end{tabular}

shown for the spatial contrast. Neither did global blood-flow measurements suggest greater diffuse activity in high-g tasks (14). Instead, the data strongly favor the hypothesis that lateral frontal functions are selectively recruited by high- $g$ tasks.

Our behavioral development work suggested a converging test of this conclusion. There was considerable variation in $g$ correlations among various candidates we investigated for the role of low- $g$ spatial control task. Although the reasons for this variation are unknown, it allowed us to select a further task for inclusion in the PET experiment whose $g$ correlation was high despite a simple physical-match format and no strong element of problem solving [Fig. 1C; for behavioral data see Table 1 and (15)]. The new task was compared with our standard low- $g$ spatial control, to which it was identical except

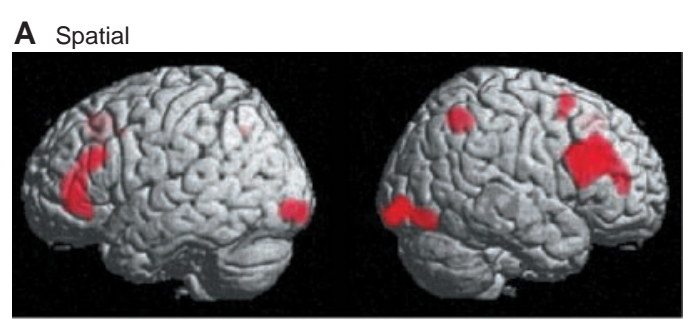

Fig. 2. Significant activations for three contrasts, rendered onto canonical T1-weighted brain image of SPM99. (A) Spatial high- $g$ minus spatial low- $g$ $(P<0.05$ corrected for multiple comparisons). (B) Verbal high- $g$ minus verbal low- $g$ ( $P<0.05$ corrected). (C) Circles minus spatial low- $g(P<0.001$ uncorrected).

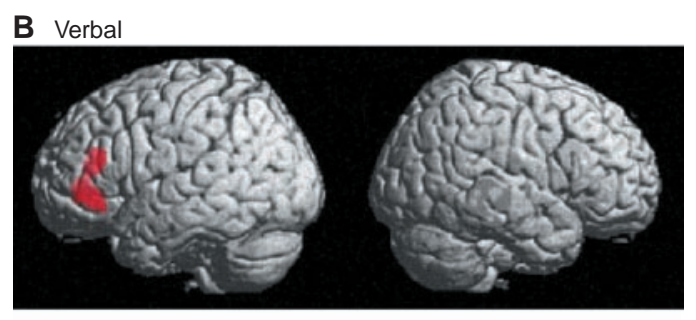

\section{Circles}

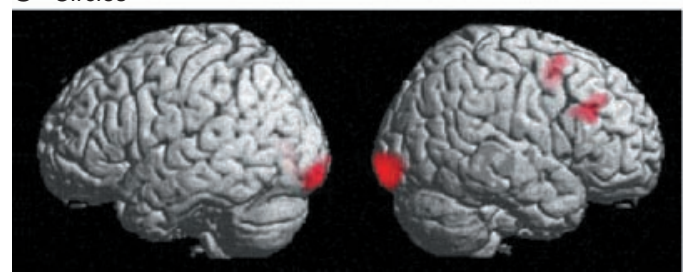

for the exact shapes used in the stimulus array. At the threshold of $P<0.05$ corrected for multiple comparisons, there were no frontal differences between these two tasks. At the less conservative threshold of $P<0.001$ uncorrected, however, the higher $g$ task was associated with lateral frontal activation in the right hemisphere (Fig. 2C and Table 2), closely corresponding to the similar activation in our main spatial contrast.

Evidently, a neural system associated with Spearman's $g$ should be recruited by many different forms of cognitive demand. In a recent analysis of imaging findings, indeed, we have shown that diverse forms of demand, including task novelty, response competition, working memory load, and perceptual difficulty, produce broadly similar lateral frontal activations covering a region closely similar to the frontal activations seen here (16). On the medial surface, all these demands are also associated with specific recruitment of the dorsal part of the anterior cingulate, close to the medial frontal activity seen here only for the spatial problemsolving task. If future work shows this medial activity also to be generally associated with different high- $g$ tasks, this will suggest an interpretation of $g$ in terms of a specific frontal network important in the brain's response to diverse cognitive challenges.

To show that $g$ is associated with a relatively restricted neural system is not, of course, to show that it cannot be divided into finer functional components. For the future, indeed, a central problem will be development of more detailed models of $g$ in terms of component frontal functions and their interactions. Mean- while, the almost century-long debate between rival theories of $g$ reflects the interpretational limitations of correlational data. The present data offer hope that the neural basis for $g$ may prove a more tractable problem. They suggest that $g$ reflects the function of a specific neural system, including as one major part a specific region of the lateral frontal cortex.

\section{References and Notes}

1. C. Spearman, Am. J. Psychol. 15, 201 (1904); The Abilities of Man (Macmillan, New York, 1927).

2. J. C. Raven, J. H. Court, J. Raven, Manual for Raven's Progressive Matrices and Vocabulary Scales (Lewis, London, 1988).

3. G. H. Thomson, Br. J. Psychol. 8, 271 (1916); The Factorial Structure of Human Ability (Univ. of London Press, London, ed. 5, 1951).

4. L. L. Thurstone, The Vectors of Mind (Univ. of Chicago Press, Chicago, IL, 1940).

5. A. R. Jensen, The g Factor (Praeger, Westport, CT, 1998); L. G. Humphreys, in Intelligence and Learning, M. P. Friedman, J. P. Das, N. O'Connor, Eds. (Plenum, New York, 1981), pp. 87-102; N. J. Mackintosh, IQ and Human Intelligence (Oxford Univ. Press, Oxford, 1998).

6. J. Duncan, Ergonomics 33, 1265 (1990); J. Duncan, P. Burgess, H. Emslie, Neuropsychologia 33, 261 (1995); J. Duncan, H. Emslie, P. Williams, R. Johnson, C. Freer, Cogn. Psychol. 30, 257 (1996); see also R. W. Engle, M. J. Kane, S. W. Tuholski, in Models of Working Memory: Mechanisms of Active Maintenance and Executive Control, A. Miyake and P. Shah, Eds. (Cambridge Univ. Press, Cambridge, 1999), pp. 102-134.

7. A. D. Baddeley, Working Memory (Oxford Univ. Press, Oxford, 1986); M. Petrides, in Handbook of Neuropsychology, F. Boller and J. Grafman, Eds. (Elsevier, Amsterdam, 1994), vol. 9, pp. 59-82; T. Shallice and P. W. Burgess, Brain 114, 727 (1991).

8. B. Marshalek, D. F. Lohman, R. E. Snow, Intelligence 7, 107 (1983).

9. Studies were designed to measure correlations between a range of candidate tasks for PET and standard measures of $g$. In the first study, 60 participants were tested, mean age 42 (range 29 to 51 ). In three test 
sessions, participants completed a range of tasks, including high- and low- $g$ spatial (Fig. 1A); high- and low- $g$ verbal (Fig. 1B); and two standard measures of $g$, Cattell's Culture Fair, Scale 2 Form B (17) (mean score in our sample 34/46 items correct, range 24 to 43 ), and the verbal scale of the AH4 (18) (mean score in our sample 43/65 items correct, range 27 to 58 ). To obtain an overall measure of $g$ for each participant, scores on the latter pair of tasks were standardized and averaged. Further data on the two verbal tasks, again completed with a range of other tasks, were obtained in a second study of 46 participants, mean age 42 (range 35 to 51). In this study, the measure of $g$ was Cattell's Culture Fair, Scale 2 Form A, with one section omitted because of overlap with other tasks in the study (mean score in our sample 24/34 items correct, range 12 to 31). For the spatial and verbal tasks, overall mean scores (number correct in $4 \mathrm{~min}$ ) were as follows: high-g spatial, 12 (range 4 to 18); low- $g$ spatial, 198 (range 162 to 225); high- $g$ verbal, 7 (range 1 to 17); low- $g$ verbal, 41 (range 16 to 75 ).

10. PET was used to obtain regional cerebral bloodflow ( $\mathrm{CCBF}$ ) data in 13 right-handed participants, mean age 26 (range 21 to 34). PET imaging was approved by the Ethics Committee of Heinrich-Heine-University Düsseldorf (Application 868). Measurements were made with an eight-ring PET camera (PC4096 plus, GE/Scanditronix, Uppsala, Sweden). The session included six scans (one for each of the tasks described in this report, plus one more of no relevance here), with the order of tasks approximately counterbalanced across participants. To allow for decay of radiation (19), successive scans were separated by 15 min, toward the end of which the 0.5 -min practice period (see legend to Fig. 1) of the next task was given. For each scan, $1480 \mathrm{MBq}$ of $\left[{ }^{15} \mathrm{O}\right]$ butanol was injected into the right brachial vein, flushed with 10 $\mathrm{ml}$ of saline. PET scanning and the 2-min task period (see legend to Fig. 1) began at the moment of the injection, so that the participant was fully engaged in the task when radioactivity reached the brain about $11 \mathrm{~s}$ later. As previously described (20), rCBF was calculated from the first $40 \mathrm{~s}$ of dynamically recorded head uptake data.

11. PET image slices were reconstructed with a Hanning filter to an effective image resolution (full width at half maximum, FWHM) of $9 \mathrm{~mm}$ with a slice thickness of $6.4 \mathrm{~mm}$. Further analysis was undertaken with SPM99 (www.fil.ion.ucl.ac.uk/spm). Scans for each subject were realigned, spatially normalized onto the PET template, and smoothed with an isotropic Gaussian kernel with FWHM set at $16 \mathrm{~mm}$. The SPM99 gray matter threshold was set to its default value. For task comparisons, an ANCOVA (analysis of covariance) model was fitted to the data for each voxel. To remove confounds due to head movement across scans (21), we tested $F$-value images to determine whether scan order or any of the six head movement parameters were significantly associated with $\mathrm{rCBF}$ values (22). Those parameters with significant associations (scan order, translation in $z$, rotation in $x$ ) were set as covariates of no interest, along with global blood flow. In this report we describe only relative increases in rCBF (activations) in high- $g$ tasks. For the spatial contrast only, there were also significant deactivations (high- $g<$ low- $g$ ) in regions of motor and medial premotor cortex, reflecting the higher response rate in the low- $g$ task, and bilaterally at the temporoparietal junction.

12. M. Corbetta, F. M. Miezin, G. L. Shulman, S. E. Petersen, J. Neurosci. 13, 1202 (1993); S. M. Courtney, L. Petit, J. Ma. Maisog, L. G. Ungerleider, J. V. Haxby, Science 279, 1347 (1998); E. Wojciulik and N. Kanwisher, Neuron 23, 747 (1999).

13. G. Esposito, B. S. Kirkby, J. D. Van Horn, T. M. Ellmore, K. F. Berman, Brain 122, 963 (1999); V. Prabhakaran, J. A. L. Smith, J. E. Desmond, G. H. Glover, J. D. E. Gabrieli, Cogn. Psychol. 33, 43 (1997).

14. Mean values of estimated global blood flow ( $\mathrm{ml} \mathrm{dl}^{-1}$ $\min ^{-1}$ ) for each task were as follows: high- $g$ spatial, 49.4; low- $g$ spatial, 49.9; high- $g$ verbal, 49.8; low- $g$ verbal, 49.7

15. The circles task was included in the first behavioral study (9) and the PET session (10). In the behavioral study, mean score (number correct in $4 \mathrm{~min}$ ) was 87 (range 53 to 112). In PET, the mean estimated global blood flow ( $\mathrm{ml} \mathrm{dl} \mathrm{Cl}^{-1} \mathrm{~min}^{-1}$ ) was 49.4 .

16. J. Duncan and A. M. Owen, Trends Neurosci., in press.

17. Institute for Personality and Ability Testing, Measuring Intelligence with the Culture Fair Tests (The Institute for Personality and Ability Testing, Champaign, IL, 1973).

18. A. W. Heim, AH4: Group Test of General Intelligence (NFER-Nelson, Windsor, UK, 1970).

19. H. Herzog et al., Eur. J. Nucl. Med. 21, 138 (1994).

20. H. Herzog, R. J. Seitz, L. Tellmann, H.-W. MüllerGärtner, J. Cereb. Bloodflow Metab. 16, 645 (1996)
21. M. Brett, P. Bloomfield, D. J. Brooks, J. F. Stein, P. M. Grasby, Neuroimage 9, S56 (1999).

22. K. J. Worsley, J.-B. Poline, K. J. Friston, A. C. Evans, Neuroimage 6, 305 (1997).

23. J. Talairach and P. Tournoux, Co-Planar Stereotaxic Atlas of the Human Brain (Thieme, New York, 1988).

24. R. B. Ekstrom, J. W. French, H. H. Harmon, D. Derman, ETS Kit of Factor-Referenced Cognitive Tests (Educational Testing Service, Princeton, NJ, 1976).

25. W. Wothke et al., Rep. No. AFHRL-TR-90-67 (Air Force Human Resources Laboratory, Brooks Air Force Base, TX, 1991).

10 March 2000; accepted 26 May 2000

\title{
The Role of GTP-Binding Protein Activity in Fast Central Synaptic Transmission
}

\author{
Tomoyuki Takahashi,* Tetsuya Hori, Yoshinao Kajikawa, \\ Tetsuhiro Tsujimoto
}

\begin{abstract}
Guanosine $5^{\prime}$-triphosphate (GTP)-binding proteins (G proteins) are involved in exocytosis, endocytosis, and recycling of vesicles in yeast and mammalian secretory cells. However, little is known about their contribution to fast synaptic transmission. We loaded guanine nucleotide analogs directly into a giant nerve terminal in rat brainstem slices. Inhibition of G-protein activity had no effect on basal synaptic transmission, but augmented synaptic depression and significantly slowed recovery from depression. A nonhydrolyzable GTP analog blocked recovery of transmission from activity-dependent depression. Neither effect was accompanied by a change in presynaptic calcium currents. Thus, G proteins contribute to fast synaptic transmission by refilling synaptic vesicles depleted after massive exocytosis.
\end{abstract}

Fast synaptic transmission is mediated by quantal packets of neurotransmitters released from synaptic vesicles through exocytosis (1). When the synaptic vesicles in the readily releasable pool (RRP) are depleted, they are replenished through vesicle recycling from a reserve pool (2). In yeast and mammalian secretory cells, a variety of $\mathrm{G}$ proteins are involved in vesicle endocytosis, trafficking, and exocytosis (3). However, the functional role of $\mathrm{G}$ proteins in fast synaptic transmission remains unclear. Synaptic transmission can be blocked by guanine nucleotide analogs (4) or Rab3A-binding peptides (5) injected into squid giant nerve terminals, suggesting that monomeric $\mathrm{G}$ proteins such as Rab3A may contribute to exocytosis (4, $6)$. In contrast, exocytosis is inhibited by overexpression of Rab3A or Rab3 regulator proteins in secretory and hippocampal cells, suggesting that this $\mathrm{G}$ protein may negatively modulate exocytosis (7). As a step toward clarifying the individual roles of $\mathrm{G}$ proteins, we studied the overall contribution of presynaptic G-protein activity to fast synaptic transmission by infusing guanine nucleotide analogs into the giant

Department of Neurophysiology, University of Tokyo Faculty of Medicine, Tokyo 113-0033, Japan.

*To whom correspondence should be addressed. Email: ttakahas-tky@umin.ac.jp nerve terminal, the calyx of Held, visually identified in slices of rat brainstem (8). In this preparation, presynaptic $\mathrm{Ca}^{2+}$ currents $\left(I_{\mathrm{pCa}}\right)$ and glutamatergic excitatory postsynaptic currents (EPSCs) can be recorded simultaneously while drugs of given concentrations are applied into the nerve terminal through a whole-cell pipette $(9,10)$.

In paired pre- and postsynaptic recordings, EPSCs were evoked stably at $0.1 \mathrm{~Hz}$ in a postsynaptic principal cell in the medial nucleus of trapezoid body (MNTB) by presynaptic action potentials elicited at the calyx of Held (9-11). Application of guanosine 5'-O-(2thiodiphosphate) (GDP $\beta S, 3$ to $6 \mathrm{mM}$ ) into the calyx through pipette perfusion blocked G-protein activity, as indicated by a marked reduction of the baclofen-induced EPSC inhibition (12), but had no effect on EPSCs (Fig. 1A) (mean amplitude 10 min after infusion $=107 \pm 9.8 \%$ of control, $n=4$ ). Thus, presynaptic G-protein activity is not immediately required for basal synaptic transmission.

We next examined whether G-protein activity is involved in synaptic depression. EPSCs were evoked by $I_{\mathrm{pCa}}$ at $0.1 \mathrm{~Hz}(9,11)$. After a stable epoch, a train of 30 stimuli at $10 \mathrm{~Hz}$ depressed EPSC amplitude to $57 \pm 7 \%$ (mean $\pm \mathrm{SEM}, n=6$ ), whereas $I_{\mathrm{pCa}}$ was not 